\title{
LITERATUR
}

Annaheim H. 1936. Die Landschaftsformen des Luganerseegebietes. Geogr. Abhandlungen. Dritte Reihe Heft 8, Stuttgart. Saibene C. 1950. L'anfiteatro morenico del rio Faloppia (Como). Bollettino del comitato glaciologico italiano. N. I = II serie, Torino. Nangeroni G. 195t. I ter*reni pleistocenici dell'anfiteatro morenico del Verbano e del territorio varesino. Atti della Soc. It. di Sc. Nat. e del museio civico di storia nat. in Milano. Vol. 93, fasc. I-II. Milano.

\section{VERÄNDERUNGEN DES GOLFES VON THESSALONIKI}

\author{
LeONidAs Eumorphopulos
}

Wie die meisten Golfe der Erde unterlag auch derjenige von Thessaloniki in Nordgriechenland im Lauf der Zeit wesentlichen Veränderungen durch Verlandungsvorgänge. Im folgenden sei versucht, auf Grund verschiedener Untersuchungen eine Vorstellung davon zu bieten, wie diese Vorgänge sich vollzogen und welche Folgen sie für das Relief des Golfes gehabt haben.

\section{GESCHICHTLICHES}

Die historisch-geographischen Karten vom Golf von Thessaloniki zeigen, daß um 500 v. Chr. das Meer sich weit nach Westen bis an den Fuß der Gebirge erstreckte, so daß Pella, die Hauptstadt Philipps von Makedonien und Alexanders des Großen, eine Küstenstadt war. Die Stadt Skydra lag ca. $5 \mathrm{~km}$, Verria ca. $10 \mathrm{~km}$ vom Meer entfernt. Die Städte Termae und Sindos an der Ostküste dagegen waren Häfen. Die Werke der alten Schriftsteller bestätigen dies und erwähnen, daß gegenüber der Küstenstadt Pella sich die Insel Phakos befunden habe, in deren Festung die makedonischen Könige ihre Schätze aufbewahrten. Die Ruinen von Pella wurden 1959 entdeckt und sind in Ausgrabung begriffen.

Der Geologe STAnley Casson hat die Fortschritte der Zuschüttung der Westbucht des Golfes von Thessaloniki kartographisch festgehalten, wo vor allem der Fluß Axios wirkte, während an der Südküste der Haliakmon sedimentierte. Zwischen beiden Flüssen wurde Geschiebe vieler Wildbäche von den Bergen Païkos und Wermion abgelagert, wodurch um 100 v. Chr. der Westteil des Golfes von Thessaloniki abgeschnitten wurde, weil die Deltas von Axios und Haliakmon verschmolzen. Es bildete sich der Binnensee von Ludias. Da der Geschiebetransport fortdauerte, verkleinerte dieser sich und verwandelte sich 1925 in den schilfbedeckten und von Sümpfen umgebenen Süßwassersee von Jannitsa. Sein Wasserstand betrug $4,5-5,0 \mathrm{~m}$ über dem mittleren Meeresniveau, die größte Tiefe 5,o m. Die westliche Bucht war also bereits zu dieser Zeit stark zugeschüttet. Nunmehr, nach weiterer Verlandung und Setzung des Bodens um $1,5-2 \mathrm{~m} \mathrm{am}$ niedrigsten Punkt in Seemitte, ist der Boden um $1,5 \mathrm{~m}$ höher als das mittlere Meeresniveau. Das Wasser des Sees floß durch den Ludias-Fluß zum Meer. An der Ostküste fehlen größere Flüsse, und der Anthemuntos-Fluß führt nicht so viel Schwebstoffe mit, daß sie wesentlich verändert würde.

Naturgemäß hätte die andauernde Verlandung des Jannitsa-Sees dessen völliges Verschwinden zur Folge gehabt; doch wurde sie durch künstliche Eingriffe erheblich modifiziert. Im Auftrage des griechischen Staates ging die Gesellschaft «Foundation»daran, durch eine größere Reihe hydraulischer Werke die Küstenebene vor den Fluß- und Wildbachüberschwemmungen zu schützen und durch Austrocknung des Jannitza- und anderer Seen und Sümpfe Agrarland zu gewinnen. Eine der wichtigsten Arbeiten war die Schaffung eines $18 \mathrm{~km}$ langen neuen Bettes des Axios von der Brücke der Eisenbahn Thessaloniki-Athen bis zum Meer. Um künftige Überschwemmungen zu vermeiden, errichtete man parallel dazu in einem Abstand von 1 too m Dämme. Damit wurde die Stadt Thessaloniki selbst vor einer Wirtschaftskatastrophe gerettet, da sie sonst innerhalb von etwa 5o Jahren durch die Ablagerungen des Axios vom Meer getrennt worden wäre. Ein zweites großes hydraulisches Werk war die Regulierung des Haliakmon, die ebenfalls mit der Konstruktion eines (linksseitigen) Dammes verbunden war. Weiter bedeutete der Bau des Sammelkanals Wermion am Fuß des gleichnamigen Gebirges einen wirksamen Schutz der Ebene vor den Wildbächen desselben, die nun nach dem Haliakmon abgeleitet werden. Auch an der Nordseite des Jannitza-Sees wurden zahlreiche Wildbäche reguliert, deren Wasser der Ludias-Fluß und der Wardarobaschi-Kanal aufnehmen. Von den Entwässerungen ist die Trockenlegung des JannitzaSees und der benachbarten Sümpfe die wichtigste. Im ganzen veränderte sich mit diesen Werken die Situation der Thessaloniki-Ebene aufs gründlichste. Außerdem wurden neue Bedingungen für 


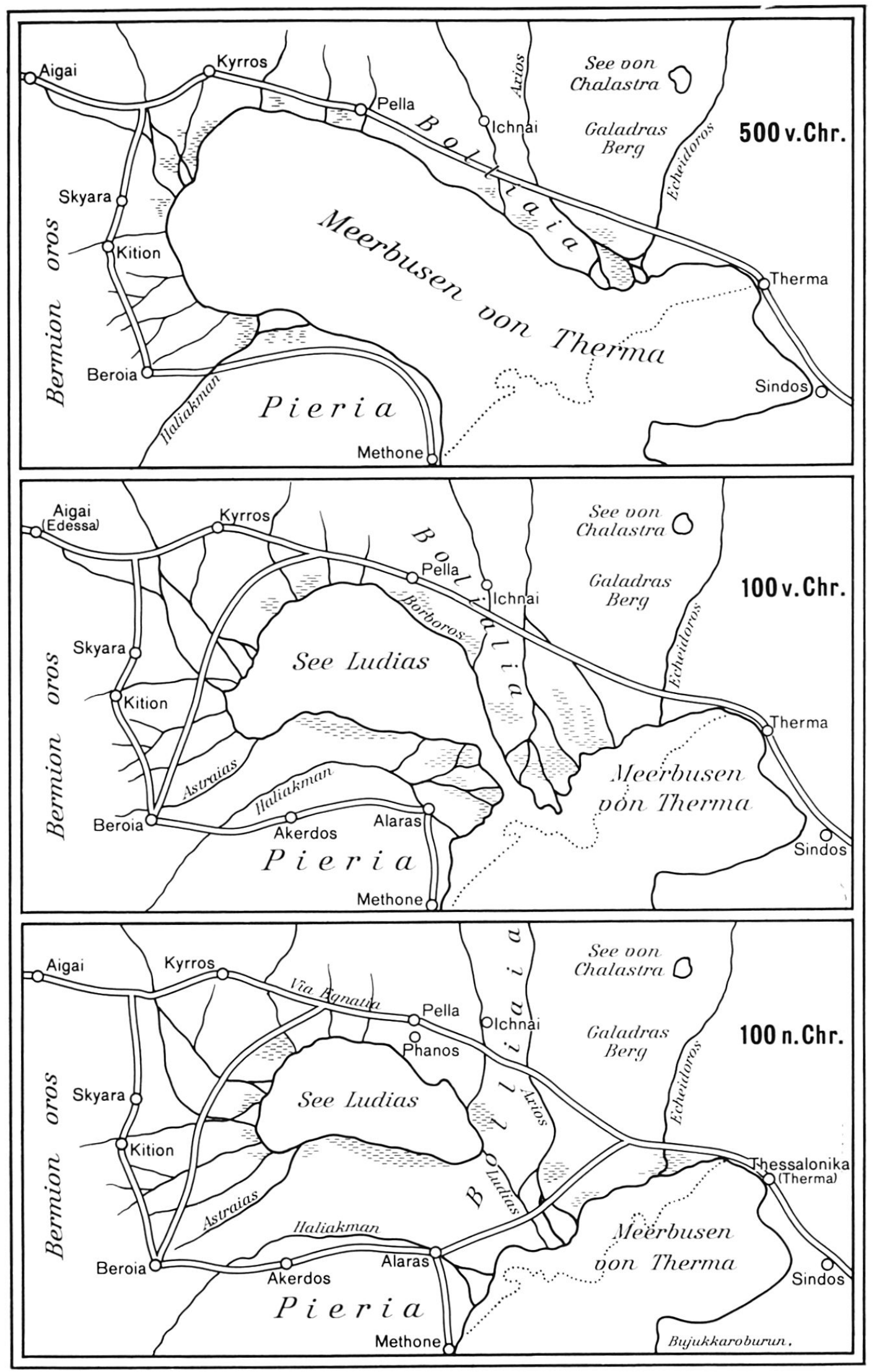

Karte 1 Antike Stadien der Verlandung

die Schwebstoffablagerung in den Golf geschaffen, was für dessen künftige Kolmatierung von größter Bedeutung sein dürfte. 


\section{DAS FLUSSGEBIET}

Das Einzugsgebiet des nördlichen Golfes von Thessaloniki hat eine Fläche von $35265 \mathrm{~km} 2$, an welchem der Abflußraum des Axios mit 67,4\%, der des Haliakmon mit 26,9\% partizipieren. Beide zusammen beanspruchen demnach einen Anteil von $9+, 2 \%$.

Tabelle 1 Abflußgebiete

Flu $B$

Haliakmon bei der Kokowa-Brücke (Stauwerk)

Sammelkanal Wermion

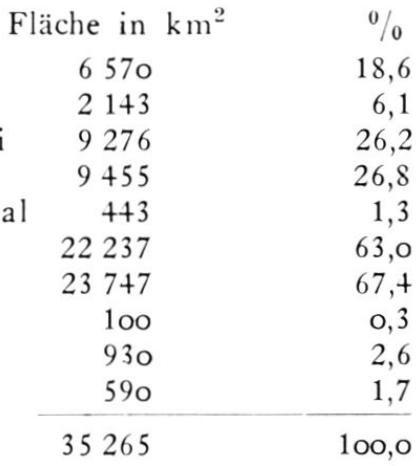

Haliakmon bei der Eisenbahnbrücke Athen-Thessaloniki

Haliakmon bei der Mündung ins Meer

Wildbäche zwischen Wermion und Wardarobaschi-Kanal

Axios an der griechisch-jugoslawischen Grenze

Axios bei der Mündung ins Meer

Das Gebiet dieser Flüsse ist gebirgig; es hat Gipfel von 2000-2200 m Höhe, die sich über

Wildbäche zwischen Axios und Gallicos

Gallicos

Anthemus und kleine Bäche an der Ostküste des Golfes

Gesamtgebiet

8,6

6,1

26,8

1,3

63,0

2,6

oo,o Ausläufern der Dinarischen Alpen erheben. Eigentliche Ebenen oder Hochplateaus sind nur klein; die Flüsse Axios und Haliakmon sowie ihre Tributäre fließen meist durch schmale tiefe Täler mit großem Gefälle. Beim Kastoria-See liegt der Lauf des Haliakmon in 63o m Höhe, während der Axios bei Skopje nur $250 \mathrm{~m}$ erreicht. Vom Einzugsgebiet des ersteren ist ein Drittel mit buschartigen Wäldern bedeckt, der Rest besteht aus Weiden und Feldern; doch gibt es auch gänzlich kahle Berge. Im Einzugsgebiet des Axios sind die Bedingungen etwas günstiger. Die mittleren jährlichen Regenmengen dieses Gebietes betragen $650 \mathrm{~mm}$, während sie im Einzugsgebiet des Haliakmon bei 850 , in dem des Wermion-Kanals bei $900 \mathrm{~mm}$ liegen. Sturzregen sind häufig, besonders im Sommer. Diese meteorologischen Bedingungen begünstigen intensive Erosion, die in gewissen Gebieten so groß ist, daß der Volksausdruck «die Felsen wachsen» entstand, weil der «weiche» Boden so rasch abgetragen wird, daß die Berge ständig höher zu werden scheinen.

\section{HYDROLOGIE}

Aus Tabelle 2 ist ersichtlich, daß der Haliakmon, dessen Einzugsgebiet fast 2,5mal kleiner ist als dasjenige des Axios, den annähernd gleichen mittleren Abfluß hat wie der Axios und auch dieselbe Abflußmenge, d. h. über $4 \mathrm{~km}^{3}$ dem Meere zuführt. Dies ist einerseits aus der geringern Regenmenge und andererseits aus der größern Versickerung im Axios-Gebiet zu erklären.

Tabelle 2

Fluß Axios an der Eisenbahnbrücke Saloniki-Belgrad

Monat

Januar
Februar
März
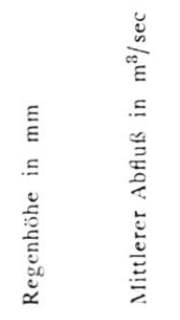

139

$52 \quad 177$

$70 \quad 210$

$65 \quad 280$

$65 \quad 237$

$5+\quad 127$

$2+\quad 51$

$32 \quad 35$

to 37

$60 \quad 4+$

$65 \quad 85$

$650 \quad 130 \quad 4086720000$

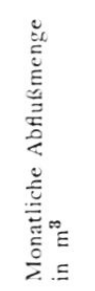

372297600

428198 too

562464000

725760000

$63+480800$

$32918+000$

136598 too

93744000

95 9ot ooo

117849600

220320000

369619200
+086720000

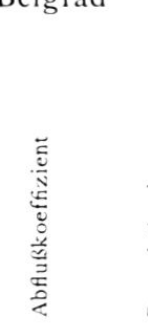

0,35

0,37

0,36

0,50

$0,+3$

0,27

0,25

0,13

0,11

0,09

0,15

$0,22 \quad 115$

$0,28 \quad 850$
Fluß Haliakmon am Derivationswehr (Kokowa-Brücke) 
Sammelgraben Wermion

\begin{tabular}{|c|c|c|c|c|c|c|c|c|}
\hline Monat & 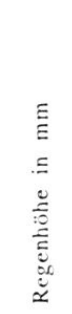 & 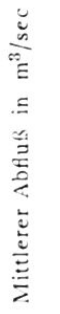 & 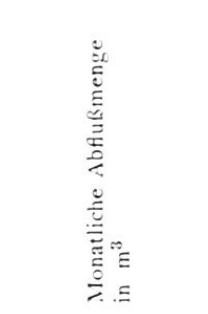 & 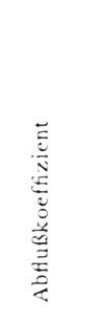 & 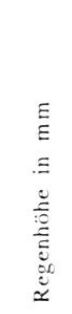 & 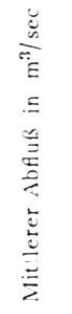 & 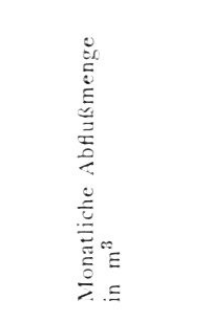 & 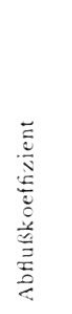 \\
\hline Januar & $9+$ & 36 & $96+22$ too & 0,48 & 72 & $1+7$ & $39372+000$ & 0,59 \\
\hline Februar & 56 & +3 & $10+025600$ & 0,87 & 67 & 190 & $+596+8000$ & $0,7+$ \\
\hline März & 86 & +7 & $12588+800$ & 0,68 & 70 & 221 & 591926 too & 0,91 \\
\hline April & 78 & 53 & 137376000 & 0,82 & 75 & 212 & $5+950+000$ & 0,78 \\
\hline Mai & 84 & 47 & $12588+800$ & 0,70 & 73 & 180 & 482112000 & 0,71 \\
\hline Juni & 88 & $3+$ & 88128000 & 0,47 & 68 & 116 & 300672000 & $0,+7$ \\
\hline Juli & 50 & 23 & 61603200 & 0,58 & $4+$ & 62 & 166060800 & $0,+1$ \\
\hline August & $2+$ & 17 & 45532800 & 0,89 & 30 & $4+$ & $1178+9600$ & $0,+2$ \\
\hline September & 52 & 18 & 46656000 & $0,+2$ & $6+$ & +1 & 106272000 & 0,18 \\
\hline Oktober & 88 & 26 & 69638 too & 0,37 & 93 & 61 & 163382 too & 0,19 \\
\hline November & 102 & 29 & 75168000 & 0,34 & 93 & 96 & 248832000 & 0,29 \\
\hline Dezember & 98 & $4+$ & $1178+9600$ & 0,56 & 111 & 203 & $5+3715200$ & 0,53 \\
\hline Jahr & 900 & 35 & $109+169600$ & 0,57 & 860 & 131 & +123699200 & 0,52 \\
\hline
\end{tabular}

Das größte Hochwasser wurde bisher am 10. Dezember 1935 beobachtet, wobei die Durchflußmenge des Axios $2450 \mathrm{~m}^{3} / \mathrm{sec}$, die des Haliakmon bei der Kokowa-Brükke $3300 \mathrm{~m}^{3} / \mathrm{sec}$, bei der Eisenbahnbrücke Athen $4040 \mathrm{~m}^{3} / \mathrm{sec}$, die des Sammelkanals Wermion $1250 \mathrm{~m}^{3} / \mathrm{sec}$ und die des Gallicos $700 \mathrm{~m}^{3} / \mathrm{sec}$ betrug. Die kleinsten Durchflußmengen registrierte man zumeist im Sommer; sie betragen $9,5-10 \mathrm{~m}^{3} / \mathrm{sec}$ bei den gröBern Flüssen, $7 \mathrm{~m}^{3} / \mathrm{sec}$ im Sammelkanal Wermion und $0,1 \mathrm{~m}^{3} / \mathrm{sec}$ im Gallicos.

\section{GESCHIEBEF ÜHRUNG}

An der griechisch-jugoslawischen Grenze bestehen die Geschiebe des Axios aus Kiesen und groben Sanden, stromabwärts schleppt der Wildbach Gorgopi kleine Mengen ebenfalls von Kiesen und Sanden in den Fluß. Die Sammelkanäle tragen nur feinen Sand und Schlamm mit sich. Ins Meer gelangen aus diesen Gebieten nur sehr feine Sande und Schlamm. Im Bett des besser bekannten Haliakmon - weil ganz auf griechischem Territorium fließend - werden von der Quelle bis zur Mündung in den Kastoria-See Geschiebe verschiedener Größe transportiert; stromabwärts vermindert sich letztere, jedoch bringen die zufließenden Wildbäche erneut verschieden grobes Geschiebe mit. Unterhalb der Kokowa-Brücke jedoch verfeinern sich die Feststoffe zusehends, sie verwandeln sich in feinen Schweb und Schlamm, die schließlich das Delta des Flusses bilden. Auch der Sammelkanal Wermion und die kleinen Wildbäche führen nur feine Sande und Schlamm, und ähnlich ließen sich im Gallicos unterhalb $12 \mathrm{~km}$ vom Meer entfernt ausschließlich solche feinen Geschiebe feststellen. Der Ludias-Fluß ist im Grunde ein 38 km langer Entwässerungskanal, der aus dem ehemaligen Jannitza-See kommt und vor allem das Wasser der kleinen Wildbäche zum Meer führt. Diese enthalten nur kleine Schwebstoff- und Geschiebemengen, und von ihnen gelangen nur wenige Prozent bis zum Meere. Alle andern fließenden Gewässer sind Zuflüsse der genannten größern und beeinflussen daher die Veränderungen der Küste höchstens mittelbar.

Da bis zur Gegenwart keine systematische Kontrolle der Geschiebeführung der Flüsse vorgenommen wurde, muß zu deren Beurteilung die sogenannte topographische Methode des Vergleichs der Querschnitte der Flüsse und des Meeres zu verschiedenen Zeitpunkten verwendet werden. 
Die Flußgebiete des Axios, Haliakmon und Gallicos. Die Mündungen der in den westlichen Thessaloniki-Golf fließenden Gewässer wurden öfters verlagert, doch haben wir davon keine konkreten Kenntnisse, weshalb zur Charakterisierung die Karten des 19. und 20. Jahrhunderts heranzuziehen sind. Zwischen 1832 und 1916 erfolgten die topographische Aufnahme des Golfes sowie die Tiefenmessungen durch die englische Kriegsmarine, deren Ergebnisse die Admiralität publizierte. Seit 1924 werden die Karten vom griechischen Ministerium aufgenommen und herausgegeben. Diesen Karten ist zu entnehmen, daß sich die Mündung des Axios um 1832 bereits nahe der heutigen befand, während er 1916 gegenüber dem Kleinen Karaburnu-Kap mündete. Um 1850 war der Ludias ein Nebenfluß des Axios; schon die nächste Karte zeigt beide unabhängig voneinander. Die Schwebstoffe dieser Flüsse werden während der Hochwasser von den Strömungen über die ganze Fläche des Golfes verteilt, so daß sein Grund den englischen Karten gemäß ganz von Schlamm bedeckt wird. Die Karte von 1916 enthält diesbezüglich die bezeichnende Bemerkung: Bei Hochwasser des Axios durchzieht eine starke Strömung den Golf bis zum Kap «Kleine Karaburnu», wo sie sich nach SW wendet, um schließlich am Kap «Große Karaburnu» vorbei in südlicher Richtung zu verschwinden. Zur Berechnung der Schwebstoffmenge des Thessaloniki-Golfes wurden die englische Karte von 1850 und die griechische von 1952 miteinander verglichen, wobei Ost-WestQuerschnitte im Abstand von $1 \mathrm{~km}$ von der Nordküste des Golfes bis Methoni (südlich des Großen Karaburnu-Kaps) konstruiert wurden. Die hieraus errechnete mittlere jährliche Absatzmenge der Geschiebe der Flüsse Axios, Haliakmon und Gallicos beträgt 1+200 $000 \mathrm{~m}^{3}$, wobei es nicht möglich war, die Menge der feinen Schwebstoffe, die südlich Methoni abgelagert werden, zu bestimmen.

Die Geschiebeführung des Gallicos ist am größten im regenreichen Winter; während des Sommers, da der Fluß nur wenig Wasser führt, ist dieses klar und beinahe frei von Feststoffen. Infolge des weiten Abstandes der Flußdämme (800-1200 m) verliert es überdies auch die Transportkraft, so daß der mitgeschleppte Sand bereits zwischen der Brücke und der Landstraße Thessaloniki-Athen sowie dem Meer zur Ablagerung kommt. Auf Grund der 1934 bis 1948 aufgenommenen Querschnitte wurde ein zwischen den Dämmen abgesetzter Feststoff́betrag von ca. $500000 \mathrm{~m}^{3}$ errechnet; die Querschnitte von 1934 und 1961 dagegen ergaben nur $123000 \mathrm{~m}^{3}$, was auf die Überschwemmung von 1960 zurückgeführt wird, die starke Erosion und damit Abtransport von Sand bewirkt hatte. Dieser ist übrigens ausgezeichnet für Bauten geeignet (insbesondere für die Herstellung von Eisenbeton), weshalb jährlich ca. $150000 \mathrm{~m}^{3}$ abgebaut und per Lastwagen nach Thessaloniki transportiert werden. Das bekannte Phänomen der Verschleppung der Ablagerungen ins Meer wurde auch bei andern Flüssen des Golfes beobachtet. Ins Meer selbst werden nach den Karten von 1850 und 1952 schätzungsweise $300000 \mathrm{~m}^{3}$ verfrachtet.

Die Ablagerungen des Axios, Haliakmon und Gallicos ins Meer sind schwer zu schätzen, da deren Mündungen nahe beieinanderliegen. Doch konnte anhand der Karte der englischen Admiralität von 1916 und der topographischen Aufnahmen der Flußmündungen von 1940 (im Maßstab 1:5000) mittels zahlreicher Querschnitte berechnet werden, daß die durchschnittliche Geschiebemenge des Axios ca. 1,5mal größer ist als diejenige des Haliakmon. Diesen Erhebungen ist zudem zu entnehmen, daß der Axios jährlich $8340000 \mathrm{~m}^{3}$, der Haliakmon $5560000 \mathrm{~m}^{3}$ und der Gallicos $300000 \mathrm{~m}^{3}$ ins Meer verlagern.

Die Geschiebe des Axios lassen sich bis ca. $10 \mathrm{~km}$ von der Mündung stromaufwärts beobachten. Während der großen Überschwemmungen treten die Wasser des Flusses über das Hauptbett und füllen oft das Überschwemmungsbett zwischen den Dämmen, wo sie ebenfalls Geschiebe absetzen. Vergleiche der Jahre 1934 und 1949 ergaben eine mittlere jährliche Ablagerungsmenge von $736000 \mathrm{~m}^{3}$. Auch in andern Abschnitten des regulierten Flußlaufs wurden Ablagerungen beobachtet; doch war es bisher nicht mög- 
lich, ihre Mengen zu bestimmen. Die gesamte jährliche Ablagerungsmenge ist zufolge der Erhebungen $9250000 \mathrm{~m}^{3}$, wobei auf Ablagerungen ins Meer $8340000 \mathrm{~m}^{3}$, ins neue Bett $736000 \mathrm{~m}^{3}$ und ins alte Bett ca. $17+000 \mathrm{~m}^{3}$ entfallen. Da der mittlere jährliche Abfluß - wie erwähnt - 4087000000 m³ beträgt, haben die Feststoffe einen Anteil von $2,26 \%$.

Die Geschiebeführung des Maliakmon zeigt ähnliche Verhältnisse. Auf Grund der Querschnitte der Jahre 1933 und 1960 wurde die jährliche Geschiebemenge auf ca. $490000 \mathrm{~m}^{3}$ berechnet. Hieraus resultieren insgesamt $6100000 \mathrm{~m}^{3}$ jährlicher Ablagerungen, die sich zu $5560000 \mathrm{~m}^{3}$ auf Absätze ins Meer, zu $490000 \mathrm{~m}^{3}$ auf solche ins Überschwemmungsbett verteilen; weitere $50000 \mathrm{~m}^{3}$ haben als «Abrundungsmenge» zu gelten.

Im Sammelkanal Wermion bestehen besondere Erscheinungen, die zu guten Teilen auf der geologischen Eigenart des Einzugsgebietes beruhen. Der Berg Wermion setzt sich vornehmlich aus Kalken und Tonschichten zusammen, und sein Osthang ist so stark bewaldet, daß nur wenig erodiert wird. Einzig der aus der Ebene an der griechischjugoslawischen Grenze stammende Fluß Moglenitsa schleppt bedeutende Sandmengen mit sich. Um den Sammelkanal vor dem Zufluß größerer Geschiebemengen zu schützen, wurden in beinahe allen Nebenbächen Sammelbecken eingebaut; das größte liegt oberhalb der Landstraße Thessaloniki-Edessa. Es wurde $193+$ und 1959 im Maßstab 1:5000 topographisch aufgenommen, und anhand der auf Grund dieser Vermessung gemachten Querschnitte errechnete man mittlere jährliche Geschiebemengen im Moglenitsa von $152000 \mathrm{~m}^{3}$, im Woda-Fluß $38000 \mathrm{~m}^{3}$. Ein Teil des Geschiebes wurde in den Sammelkanal verfrachtet und stromabwärts vom oberen Wehr abgesetzt. Während des Jahres 1949 wurden überdies Querschnitte durch den Sammelkanal im Abstand von $150 \mathrm{~m}$ aufgenommen und mit denjenigen von 1934 verglichen. Es ergaben sich folgende Geschiebemengen für die Periode 1934-1949.

Tabelle 3

\begin{tabular}{ll} 
Kilometerabschnitt & \multicolumn{1}{c}{ Abschnitt des Sammelkanals } \\
$5+500$ bis $1++900$ & $\begin{array}{l}\text { Von Wehr und Brücke } \\
\text { bis Fluß Arapitsa }\end{array}$ \\
$14+900$ bis $20+660$ & $\begin{array}{l}\text { Vom Arapits bis } \\
\text { zum Bach Skrop }\end{array}$ \\
$20+660$ bis $27+785$ & $\begin{array}{l}\text { Vom Skrop bis zum } \\
\text { Tripotamos }\end{array}$ \\
$27+785$ bis 35+260 & $\begin{array}{l}\text { Vom Tripotamos bis } \\
\text { zum Wehr }\end{array}$
\end{tabular}

\begin{tabular}{lcc} 
Herkunft der Geschiebe & $\begin{array}{c}\text { Gesamtmenge } \\
\text { in } \mathrm{m}^{3}\end{array}$ & $\begin{array}{c}\text { Mittlere jührliche } \\
\text { Menge in } \mathrm{m}^{3}\end{array}$ \\
Woda und Moglenitsa & 803235 & 58882 \\
FluB Arapitsa & $20+1+5$ & 13610 \\
Bach Skrop & 213026 & $1+202$ \\
Tripotamos & 329208 & $219+7$ \\
\hline & $162961+$ & $1086+1$
\end{tabular}

$\mathrm{Zu}$ den mitgeschleppten Geschieben muß die im Sammelkanal abgelagerte Menge, d. h. für den Moglenitsa $+1200 \mathrm{~m}^{3}$ und für den Woda $17000 \mathrm{~m}^{3}$, mitberücksichtigt werden, so daß die jährliche Geschiebemenge im Moglenitsa $193000 \mathrm{~m}^{3}$, im Woda $57000 \mathrm{~m}^{3}$ beträgt.

Auch für die kleineren Flüsse wurden Sammelkanäle gebaut; so hat der Arapitsa ein Becken von $300000 \mathrm{~m}^{3}$, das häufig durch Geschiebe gefüllt wird. Die jährliche Geschiebemenge wurde deshalb auf $35000 \mathrm{~m}$ ” berechnet. Skrop und Kutika dagegen haben keine Sammelbecken und schleppen jährlich ca. 15000 m³ Geschiebe zum Sammelkanal. Der Tripotamos, der ein Becken besitzt, dessen jährliche Geschiebemenge auf $10000 \mathrm{~m}^{3}$ geschätzt wurde, führt dem Sammelkanal ca. $300000 \mathrm{~m}^{3} \mathrm{zu}$. Die fünf Nebenflüsse des Wermion lagern somit jährlich rund $330000 \mathrm{~m}^{3}$ Geschiebe ab, die durch Bagger entfernt werden, damit der Kanal in gutem Zustande bleibt. Eine kleine Menge von Feinstoffen wird übrigens in den Haliakmon verschleppt.

Das Verhältnis der Geschiebe zur jährlichen Abflußmenge beträgt also $330000 \mathrm{~m}^{3}$ zu $1094000 \mathrm{~m}^{3}$, d.h. 0,3\%. Nunmehr ist es auch möglich, die Feststoffmengen im Ha- 
liakmon zu berechnen. Nach Tabelle 2 beträgt dessen mittlere jährliche Abflußmenge $4123700000 \mathrm{~m}^{3}$, die Ablagerungen $6100000 \mathrm{~m}^{3}$, so daß jene 1,5\% ausmachen.

Der Ludias-Fluß wurde von schwimmenden Baggern zur Austrocknung des Jannitsa-Sees ausgegraben. In diesen münden die Bäche Balitsa, Jannitsa und TschekreTschekarli; sie lagern nur geringe Schlamm- und Sandmengen ab. Stromabwärts münden in den Ludias zahlreiche Haupttrockenkanäle, welche ihm trübes Wasser zuführen. Die Sinkstoffe bilden eine nur unbedeutende Absatzmenge ins Meer; sie wird durch Tabelle 4 erfaßt :

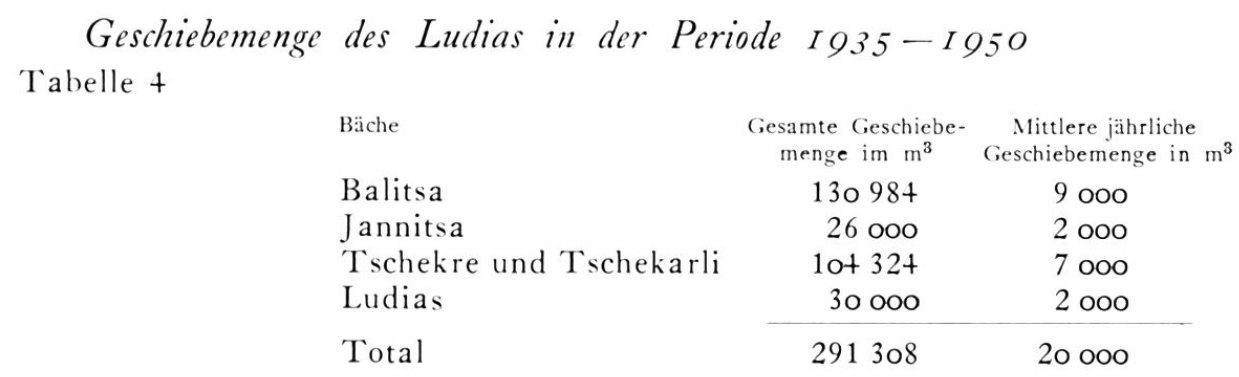

Der Sammelkanal Wardarobaschi leitet das Wasser dreier bedeutender Wildbäche dem Axios zu, deren Geschiebemengen auf Grund der Querschnitte von 1934 und 1948 auf $392000 \mathrm{~m}^{3}$ berechnet wurden, was einer jährlichen Ablagerung von $28000 \mathrm{~m}^{3}$ entspricht. Da eine kleine Menge in den Betten dieser Gewässer zurückbleibt, muß die Gesamtgeschiebemenge um $30000 \mathrm{~m}^{3}$ ergänzt werden.

$V$ on den Wildbächen zwischen Axios und Gallicos sind vier bemerkenswert, deren untere Betten reguliert wurden, wo sich folgende Geschiebemengen ablagerten:

Tabelle 5

\begin{tabular}{lrrr} 
& & Gesamtmenge in $\mathrm{m}^{3}$ & Jährliche Menge in $\mathrm{m}^{3}$ \\
Wathylakos & $1946-53$ & 69880 & 10000 \\
St. Athanassios & $1945-58$ & $28+940$ & 22000 \\
Neuer Anchialos & $19+6-58$ & 51850 & 4300 \\
Inglisch & $1947-58$ & 13800 & 1200 \\
\cline { 2 - 3 } & & & 37500
\end{tabular}

In der Thessaloniki-Ebene fließen noch verschiedene andere Wildbäche, die gleichfalls zur Verlandung des Golfes beitrugen; doch fehlen Angaben, um ihre Geschiebemengen zu berechnen, so daß wenige Bemerkungen über sie genügen müssen. Zum Beispiel wurden während der Grabung des neuen Bettes des Kryoneri in einer Tiefe von 2,5 $\mathrm{m}$ verschiedene Hausgeräte und Münzen aus der Zeit Philipps von Makedonien gefunden, wonach dieser Bach innerhalb von 2300 Jahren eine Sand- und Tonschicht von $2,5 \mathrm{~m}$ Mächtigkeit aufgeschüttet haben muß. Auch Ausgrabungen der Ruinen des alten Pella, die unmittelbar am Meer am Fuß von Hügeln aufgefunden wurden, zeigen, daß offenbar Geschiebe von 0,5 bis $0,8 \mathrm{~m}$ in deren höhern Teilen und bis 2,0 $\mathrm{m}$ in deren niemit Gipfelhöhen von 2000 m und Gefällen von 0,025 und 0,030\% liegen.

Tabelle 6 faßt sämtliche Daten zusammen, die über die Geschiebemengen gewonnen werden konnten. Darnach transportiert der Axios die größten Quantitäten; mit dem Haliakmon zusammen sind es $15350000 \mathrm{~m}^{3}$ Geschiebe. Alle andern Gewässer zusammen schleppen nur $720000 \mathrm{~m}^{3}$ mit sich, die denn auch, verglichen mit jenen, nur gering an der Verlandung des Westteils des Thessaloniki-Golfes beteiligt sind. Tabelle 6 zeigt zudem, daß die größten Geschiebemengen pro $\mathrm{km}^{2}$ durch die Wildbäche St. Athanassios und Wathylakos geliefert werden, deren Abflußgebiete in den Sand- und Tongebieten mit Gipfelhöhen von 2000 m und Gefällen von 0,025 und 0,030\% liegen. 
Tabelle 6

\begin{tabular}{|c|c|c|c|c|c|}
\hline \multirow{2}{*}{ Name des Flusses } & \multirow{2}{*}{$\begin{array}{c}\text { Fläche des } \\
\text { Abflußgebietes } \\
\text { in } \mathrm{km}^{2}\end{array}$} & \multicolumn{2}{|c|}{ (ieschiebemenge } & \multicolumn{2}{|c|}{ Geschiebemenge $\mathrm{m}^{3}, \mathrm{~km}$} \\
\hline & & $\begin{array}{l}\text { teilweise } \\
\text { in } \mathrm{m}^{3}\end{array}$ & $\begin{array}{l}\text { insgesamt } \\
\text { in } \mathrm{m}^{3}\end{array}$ & teilweise & durchschnittlich \\
\hline 1. Fluß Gallicos & 930 & 300000 & 300000 & 323 & \\
\hline 2. Fluß Axios & $237+7$ & 9250000 & 9250000 & 390 & \\
\hline 3. Fluß Haliakmon & 7312 & 6100000 & 6100000 & $83+$ & \\
\hline \multicolumn{6}{|l|}{ 4. Sammelgraben Wermion } \\
\hline Fluß Moglenitsa & 1050 & 193000 & & $18+$ & \\
\hline Fluß Wodas & 280 & 57000 & & $20+$ & \\
\hline Fluß Arapitsa & $27+$ & 35000 & & 128 & \\
\hline Bäche Skrop und Kutika & 102 & 15000 & & 147 & \\
\hline \multirow[t]{2}{*}{ Flu $B$ Tripotamos } & 228 & 30000 & & 132 & \\
\hline & $193+$ & 330000 & 330000 & & 171 \\
\hline 5. Fluß Ludias & & 2000 & & & \\
\hline Bach Balitsa & 288 & 9000 & & 31 & \\
\hline Bach Jannitsa & 46 & 2000 & & 43 & \\
\hline \multirow[t]{2}{*}{ Bach Tschekre und Tschekarli } & 88 & 7000 & & 80 & \\
\hline & 422 & 20000 & 20000 & & 47 \\
\hline 6. Sammelgraben Wardarobaschi & $2+6$ & 30000 & 30000 & 122 & 122 \\
\hline \multicolumn{6}{|l|}{ 7. Bäche zwischen Axios und Gallicos } \\
\hline Bach Wathylakos & 20 & 10000 & & 500 & \\
\hline Bach St. Athanassios & 19 & 22000 & & 1157 & \\
\hline Bach Neuer Anchialos & 18 & 4300 & & 239 & \\
\hline \multirow[t]{3}{*}{ Bach Inglisch } & 8 & 1200 & & 150 & \\
\hline & 65 & 37500 & rund to ooo & & 615 \\
\hline & $3+656$ & 16 o7o ooo & & & 463 \\
\hline
\end{tabular}

Der Haliakmon schleppt jährlich $834 \mathrm{~m}^{3}$ Geschiebe je $\mathrm{km}^{2}$; dies ist aus der bereits erwähnten Steilheit der Gehänge und der geringen Bewaldung erklärlich. Im AxiosGebiet ist die Geschiebemenge wesentlich kleiner, da auch die Erosion geringer ist. Im Sammelkanal Wermion werden sogar nur $171 \mathrm{~m}^{3} / \mathrm{je} \mathrm{km}^{2}$ transportiert, weil dort die Erosionsbedingungen noch ungünstiger sind. Das Einzugsgebiet des Ludias liegt fast völlig in der Thessaloniki-Ebene, wo geringes Gefälle und intensiver Ackerbau so gut wie keine Erosion zulassen, die deshalb nur im Bereich dreier Wildbäche auftritt, wie aus Tabelle 4 ersichtlich ist. Um feststellen zu können, ob die eruierte jährliche Geschiebeführung für die Zuschüttung des Golfes, die mit $1200 \mathrm{~km}^{2}$ innerhalb von $2400 \mathrm{Jahren}$ zu veranschlagen ist, sei folgende Berechnung angestellt: Da die ehemals größte Tiefe des Golfes unbekannt ist, während sie gegenwärtig - etwas südlich des Großen Karaburnu-Kaps - zwischen 25 und $35 \mathrm{~m}$ beträgt, nehmen wir an, daß die Durchschnittstiefe der westlichen Bucht $25 \mathrm{~m}$ ausmachte. Da ferner die mittlere Höhe der ThessalonikiEbene $7 \mathrm{~m}$ beträgt, muß die Mächtigkeit der Ablagerung $32 \mathrm{~m}$ sein. Das Volumen derselben wäre darnach $1200000000 \times 32=38400000000 \mathrm{~m}^{3}$. Die mittlere jährliche Ablagerung wurde auf $16070000 \mathrm{~m}^{3}$ berechnet, so daß innerhalb von 2400 Jahren $16070000 \times 2400=38568000000 \mathrm{~m}^{3}$ zur Ablagerung kamen, die ausreichend waren, um die Thessaloniki-Ebene entstehen zu lassen.

Die Entwicklung der Mündungen des Axios und des Haliakmon. Die Karte 2 zeigt die Mündungen der Flüsse Axios, Haliakmon und Ludias während verschiedener Epochen. Da diese ins Meer fließen, dessen Spiegel nur zwischen $-0,5 \mathrm{~m}$ und 0,5 $\mathrm{m}$ schwankt und geringe Strömungen bestanden, bildeten die mitgeschleppten Geschiebe vielarmige Deltas. Für die Periode 1933-1960 lassen sich aus dem Kartenvergleich folgende Schlüsse ziehen:

Das Delta des neuen Bettes des Axios, das seit 1934 benutzt wird, wächst nach Süden und befand sich im Sommer $19593600 \mathrm{~m}$ vom Ende des linken Dammes entfernt. Es bewegte sich somit mit einer jährlichen Geschwindigkeit von $114 \mathrm{~m}$. Das Delta des 


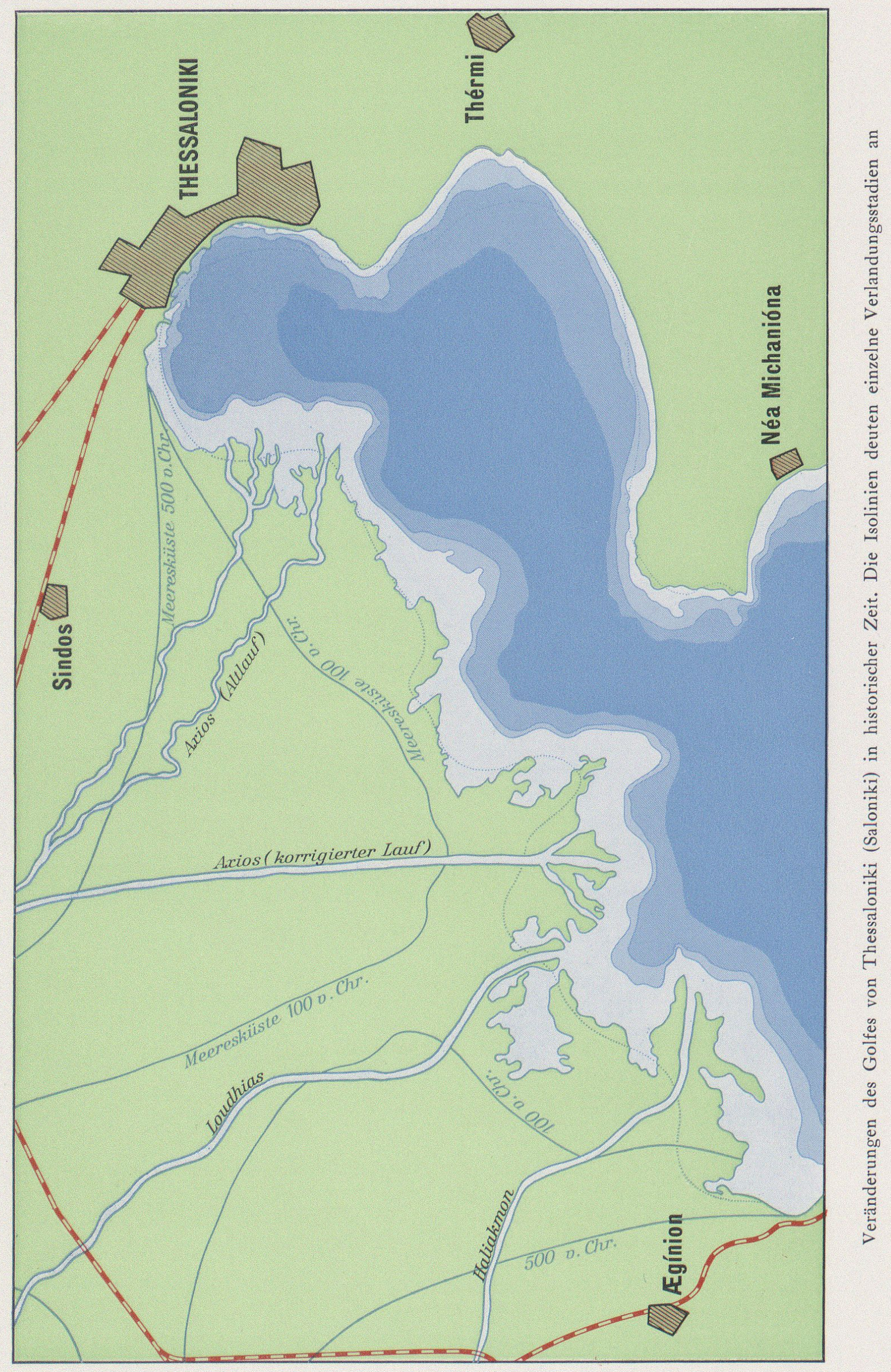


Haliakmon dehnte sich während derselben Zeit um ca.1000 m nach Südosten aus, die jährliche Wachstumsgeschwindigkeit betrug somit nur $40 \mathrm{~m}$, weil das Delta gleichzeitig nach Süden wuchs. Die Entfernung der Flußmündungen, die $19336000 \mathrm{~m}$ betrug, hat sich inzwischen auf $4000 \mathrm{~m}$ vermindert, so daß die Mündungen sich mit einer jährlichen Geschwindigkeit von $80 \mathrm{~m}$ näherten. In der Tiefe des Meeres lassen Luftaufnahmen bereits eine Begegnung der Ablagerungen erkennen, so daß die gänzliche AbschlieBung der Bucht innerhalb von 50 Jahren zu erwarten ist, wenn im Flußlauf keine Änderungen eintreten. Die Bucht wird sich dann in eine Lagune verwandeln, die von Jahr zu Jahr seichter werden wird, um schließlich durch Kolmatierung in Agrarland umgeformt zu werden.

Die Mündung des Ludias blieb während der beobachteten Zeit unverändert, da er auch während Hochwasser nur wenig Feststoffe führt. Um den Abfluß des Wassers zum Meer zu sichern, muß sein Bett allerdings erweitert und in gutem Zustande erhalten werden. Da der Ludias $5 \mathrm{~m}$ tief und bis zur Thessaloniki-Ebene schiffbar ist und die Geschiebe des Südwestarms des Axios dessen Verlängerung ins Meer öfters verschütteten, wurde der rechte Damm des Axios bis zu dessen Ende verlängert und zudem ein neuer Arm vom Beginn des Südostarmes in Südostrichtung bis zum Meer gegraben. Dieser Durchstich funktionierte während einiger Jahre gut, verursachte indes die Versandung des Südostarmes und wurde auf die Dauer selbst verschüttet, so daß der Axios wie früher durch das Hauptbett nordöstlich zum Meer fließt.

Die Entfernung von der Mündung des Axios bis zum Großen Karaburnu-Kap hat sich seit 1934 kaum verändert. Sollte sich aber das Delta in Südostrichtung entwickeln, so entsteht die Gefahr einer langsamen Versandung des Meeresdurchganges zwischen Mündung und Kap mit katastrophalen Folgen für die Schiffahrt. Es ist jedoch damit zu rechnen, daß rechtzeitig entsprechende Maßnahmen getroffen werden.

\section{DIE VERWENDUNG DER GESCHIEBE}

Die beträchtlichen Geschiebe des Axios und Haliakmon lassen sich zur systematischen Aufschüttung und Hebung der niedrigen Küstenebenen und Lagunen verwenden. Damit können bedeutende Landflächen gewonnen werden. Es genügt dazu, an jenen Stellen Flußdurchstiche anzulegen, wo man die Ablagerung der Geschiebe wünscht. Wenn dort genügend Land gewonnen ist, können die Durchstiche wieder geschlossen und andere geöffnet werden. Diese Arbeit gestattet, wenn sie von Methondi bis zur alten Mündung des Axios auf eine Länge von $25 \mathrm{~km}$ durchgeführt wird, dem Meer $75 \mathrm{~km}^{2}$ Land abzugewinnen und innerhalb von etwa 50 Jahren eine Erhöhung der Küstenzone über mehr als $100 \mathrm{~km}^{2}$ vorzunehmen, wobei nur geringe Investitionen nötig sind. Der Mensch kann somit auf wissenschaftlicher Basis die früheren Wildflüsse Mazedoniens durchaus bändigen.

\section{CHANGEMENT DE LA CONFIGURATION DU GOLFE DE SALONIQUE}

Les fleuves et rivières de la Macédonie qui se déversent dans la mer d'Egée amènent des alluvions considérables et provoquèrent déjà dans l'antiquité des inondations périodiques. La Macédonie engendre dans le sud la plaine de Salonique. La grande fertilité de cette dernière contrée, surtout pour la culture des céréales et du tabac, justifiait depuis longtemps des efforts pour les aménagements des eaux. D’autre part, les fleuves Axios et Haliakmon risquaient de bloquer un jour la baie de Salonique. La situation politique instable dans le siècle passé n'encourageait guère les occupants de faire des dépenses notables pour la correction de ces eaux. L'Etat de Grèce a néanmoins entrepris, dans les dernières années, à ce sujet des travaux considérables. L'Axios fut canalisé et son lit a été déplacé vers l'ouest hors de la baie. L'Axios déverse maintenant ces alluvions annuelles de $9000000 \mathrm{~m}^{3}$ à une place qui ne met plus en danger la navigation. Il faut bien noter que le bord de la mer a été reculé depuis les temps d'Alexandre le Grand de plus de $20 \mathrm{~km}$. L'ancienne ville d'Alexandre le Grand, Pella qui se trouvait dans ce temps au bord de la mer, est aujourd'hui à plus de $20 \mathrm{~km}$ de distance de la rive maritime. L'auteur, Ing. L. Eumorphopulos, qui étudiait dans le temps à l'Ecole Polytechnique de Zurich, a participé en majeure partie à ces grands travaux d'aménagement. 